\title{
Non-Linear Denoising of Images using Wavelet Transform
}

\author{
Ch. Mohan Sai Kumar \\ Assistant Professor \\ Dept. of ECE \\ Dhanekula Institute of \\ Engineering \& Technology
}

\author{
K. Suresh Babu \\ Assistant Professor \\ Dept. of ECE \\ Dhanekula Institute of \\ Engineering \& Technology
}

\begin{abstract}
In the present communication system, digital images can represent most of the Visual information efficiently. In the process of communication images are generally corrupted during coding, transmission and reception. The noise presence during image acquisition results in faulty analysis of the images. This faulty analysis leads to incorrect restoration of original image. Hence, image denoising should be perfectly performed to improve the quality of image for more precise diagnosis. Wavelet based shrinkage denoising will best restore the Visual content from noisy data. A new thresholding function for image denoising is proposed in this research paper. This proposed function is applied on the additive white Gaussian noise corrupted images using VISU, false discovery rate and translation invariant shrinkage rules. Performance of this new method is compared with existing hard, soft and SCAD thresholding functions using feature measure parameters like root mean square error (RMSE) and peak signal to noise ratio (PSNR). From the analysis, the new limiting function has a superior performance than all other existing thresholding functions in VISU, false discovery rate and translation invariant methods.
\end{abstract}

\section{Keywords}

Image acquisition, wavelet based shrinkage denoising, new thresholding function, additive white Gaussian noise, root mean square error (RMSE) and peak signal to noise ratio (PSNR).

\section{INTRODUCTION}

In modern days, Image processing forms core research area within the field of engineering and computer science too. The major concern in digital images is the introduction of noise in the image databases collected by the image acquisition devices, errors in the transmission medium, reproduction, compression and storage. Noise in the binary images corrupts the colour information and brightness in image. It adds irrelevant information to the image. So noise in the images should be eliminated for accurate analysis. Removal of noise is an essential step of any digital image processing system. It involves evaluation of signal degradation and restoring the original signal sufficiently with its necessary features preserved intact. Hence denoising is the initial action to be taken before performing the examination on images further. The main issue in noise removal is to preserve very fine details in the image like edges, colour information etc., Every noise removal algorithm has its own requirements, advantages and limitations [1].

Denoising and smoothing are different aspects of image processing. Smoothing removes the high frequency components and remains low frequency components. Whereas denoising removes noise and retain the signal regardless of the spectral content of the noisy signal.

The process of removing the noise in very large amount of wavelet coefficients is called as wavelet based shrinkage denoising. It is one of the mostly used denoising techniques for 1-d and 2-d images. In this research paper, we recommend a new technique of thresholding filter function for removing the additive white Gaussian noise, corrupting the original images. The performance of new thresholding function is compared with the existing hard, soft and SCAD thresholding functions by using wavelet shrinkage methods like VISU, false discovery rate and translational invariant methods [2].

\section{WAVELET SHRINKAGE DENOISING}

In wavelet shrinkage based denoising of images as in [3], the first step is to apply discrete wavelet transform to the image corrupted with noise to obtain the noisy wavelet coefficients. The resulting noisy wavelet coefficients are modified using new thresholding function with respect to the threshold calculated value using VISU, false discovery rate and translation invariant thresholding methods. After modification of wavelet coefficients, inverse wavelet transform is applied on modified wavelet coefficients to get the denoised images as in [4].

\section{THRESHOLDING TECHNIQUES}

The concept of thresholding as in [5] in wavelet transform domain tries to remove noise present in the noise degraded image due to the property of energy compaction provided of wavelet transform. According to this property, majority of the original signal power lies in the top LL band of wavelet decomposition, at the same time the noise power is scattered all over the other detail sub bands. Hence wavelet decomposition can be used to segregate the actual signal content from the noisy content. This type of isolation process eases as long as the signal power is much larger than the noise power.

\section{The mostly used thresholding techniques are}

\subsection{VISU Method}

VISU shrinkage thresholding was first proposed by Donoho and Johnstone as in [6] to assist the rule of hard thresholding. In this method, the threshold value is calculated from high pass components of detailed wavelet coefficients at the initial level of wavelet transform. In VISU method, the threshold value is directly proportional to the noise variance. In denoising of images, VISU shrink deals effectively with additive white Gaussian noise to produce a large threshold value than other complementary methods to give an excessively smooth reconstructed estimate of the original image. The main drawback with this method is that it is 
unable to remove salt $\&$ pepper noise and the minimum mean square error will also be very high. VISU Shrink greatly depends on size of the image but not on content within it. In VISU Shrink method the threshold can be estimated as

$$
\begin{gathered}
T_{V}=\hat{\sigma} \sqrt{2 \log N} \\
\hat{\sigma}^{2}=\left[\frac{\operatorname{median}\left(\left|w_{J-1, k}-\operatorname{median}\left(w_{J-1, k}\right)\right|\right)}{0.6475}\right]^{2}
\end{gathered}
$$

Where $\hat{\sigma}$ is estimated noise variance based on the median absolute deviation (MAD), a robust estimator and $\mathrm{N}$ is the number of pixels in the original image.

\subsection{False Discovery Rate Method}

The minimizing false discovery rate (min FDR) method was first initiated by B. Vidakovic as in [7] for 1-Dimenional data. It is an accepted proportion of false discoveries among the discarded null hypotheses. FDR preserves the identical threshold value for all the thresholding functions by ensuring the likely value of the small proportion of detailed coefficients incorrectly incorporated in the reconstruction of wavelet coefficients below a given fraction $\alpha$. Given the $\mathrm{L}$ detailed coefficients $\left(e_{n}, n=1,2 \ldots L\right)$, first it computes $p$-values.

$$
\mathrm{p}_{\mathrm{n}}=2\left[1-\delta\left(\left|\mathrm{e}_{\mathrm{n}}\right| / \sigma\right)\right]
$$

Where $\delta$ (.) is the cumulative distribution function and $\sigma$ is an standard deviation. Then $\mathrm{p}_{\mathrm{n}}$ values are ordered as $\mathrm{p}_{(1)} \leq \mathrm{p}_{(2)} \leq \mathrm{p}_{(3)} \ldots \leq \mathrm{p}_{(\mathrm{L})}$. From $\mathrm{n}=1$, let $\mathrm{k}$ be the major index value then

$$
\mathrm{p}_{(\mathrm{k})} \leq \frac{k}{L} \alpha
$$

The threshold value is obtained as

$$
\lambda=\sigma \delta^{-1}\left(1-\left(\mathrm{p}_{(\mathrm{k})} / 2\right)\right)
$$

\subsection{Translation Invariant Method}

Donoho and Coifman have first proposed the translation invariant method. It consists of carrying out shrinkage on each basis and taking the average of the obtained de-noised signals. There are two challenging effects present for improved detection of singularities due to taking into account all the shifts in the analysis and an effect of more powerful smoothing due to averaging of the de-noised signals on each basis. This method can get better compensation of the edges and it is also used for estimation of images as in [8].

\section{THRESHOLDING FILTERS}

After the calculation of threshold value for each sub band (LL, LH, HL and HH) except the low pass or approximation sub band, apply thresholding filter to each noisy wavelet coefficient given below, by substituting the calculated threshold value.

Thresholding filters can be used for applying the calculated threshold value on the noisy image. In this paper existing Hard, Soft and SCAD filters are considered along with hybrid thresholding filter.

Donoho and Johnstone proposed a widely recognized filter known as hard thresholding filter. Hard thresholding can also be treated as remain or destroy method or gating. The transfer function of the same is shown in figure 1. Hard thresholding is very sensitive and it has very large variance. In this method, threshold value is fixed by adopting some existing threshold rules. Hard thresholding set the value of wavelet coefficients less than or equal to the threshold to zero. The coefficients greater than this threshold value are remained same as in [9].
The hard thresholding hard $(\mathrm{W}, \lambda)$ is denoted as

$$
\begin{aligned}
\operatorname{hard}(W, \lambda) & =W & & \text { for }|W|>\lambda \\
& =0 & & \text { otherwise }
\end{aligned}
$$

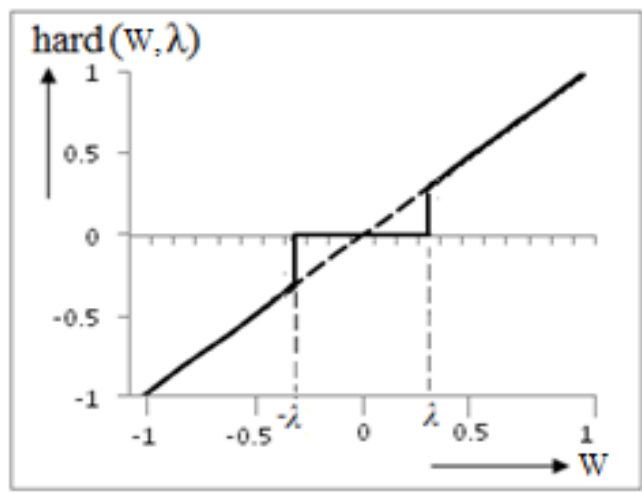

Fig 1: Hard Thresholding Filter

The soft threshold function as in [10] takes the argument and shrinks it toward zero by the threshold T. Soft thresholding filter is used to analyze the performance of denoising procedure for different levels of DWT decomposition, because the soft thresholding filter achieves near-optimal mini-max rate over a large range of Besov spaces and for the generalized Gaussian noise, the soft-thresholding filter yields a minor risk than the hard-thresholding estimator. In practice, the soft-thresholding method reclaims more regretted VISUally pleasing images over hard-thresholding since the latter one is sporadic and yields abrupt artifacts in the improved images, especially when the noise energy is more significant. Soft filter sets the coefficient value as the difference between coefficient value and threshold value if it is greater than the threshold value; otherwise the coefficient value is zero. The transfer function of soft thresholding soft $(\mathrm{W}, \lambda)$ is given below and the same is shown in the figure 2 .

$$
\begin{aligned}
\operatorname{soft}(W, \lambda) & =[\operatorname{sign}(W)](|W|-t) & & \text { for }|W|>t \\
& =0 & & \text { otherwise }
\end{aligned}
$$

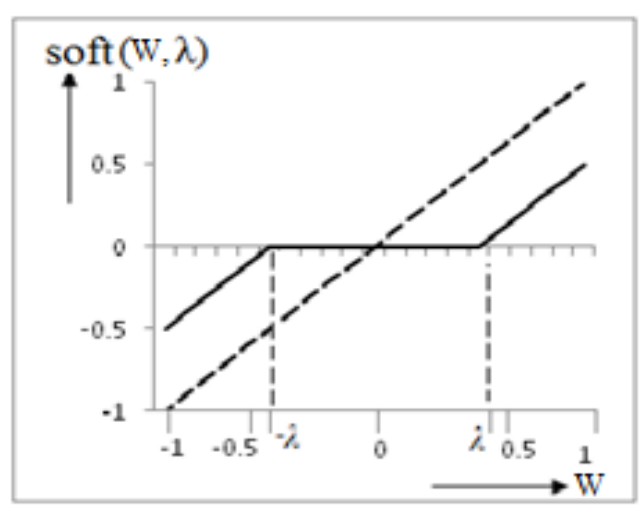

Fig 2: Soft Thresholding Filter

SCAD, smoothly clipped absolute deviation, is a piecewise linear function and is continuously differentiable in $(-\infty, 0) \mathrm{u}$ $(0, \infty)$. The SCAD derivatives are zero outside the $[-\alpha \lambda, \alpha \lambda]$. This leads to large coefficients retain as it is, while small coefficients are made zero and some other coefficients have been made shrunk towards zero. It does not create excessive bias when the wavelet coefficients are large and it produces a set of sparse solutions. The transfer function for the SCAD thresholding function is shown in figure 3 . 
The SCAD function is denoted as

$S C A D(W, \lambda)=\left\{\begin{array}{lr}\operatorname{sign}(W) \max (0,|W|-\lambda) & \text { if }|W| \leq 2 \lambda \\ (\alpha-1) W-\alpha \lambda \operatorname{sign}(W) \text { if } 2 \lambda \leq|W|<\alpha \lambda \\ W & \text { if }|W|>\alpha \lambda\end{array}\right.$

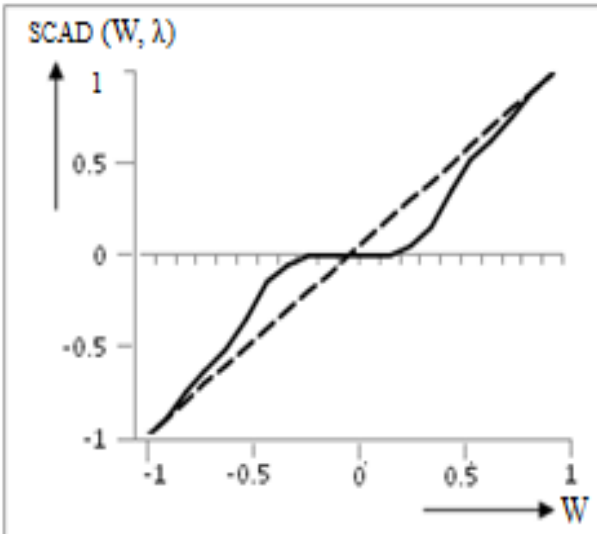

Fig 3: SCAD Thresholding Filter

\section{NOVEL THRESHOLDING FILTERS}

In this paper a novel thresholding filter function is proposed for modifying the noisy wavelet coefficients. This filter function is designed by subtracting the fraction of the noisy coefficient from the hard thresholding filter for more than threshold value. $25 \%$ of detailed coefficient value is considered for less than the threshold value. This is given as

$$
\begin{aligned}
N(W, \lambda) & =\operatorname{hard}(W, \lambda)-\left(\frac{1}{1+e^{(2 W / \lambda)}}\right) \text { for all }|W|>\lambda \\
& =0.25 * W \quad \text { otherwise }
\end{aligned}
$$

Here, $W$ represents wavelet coefficients and $\lambda$ represents threshold value.

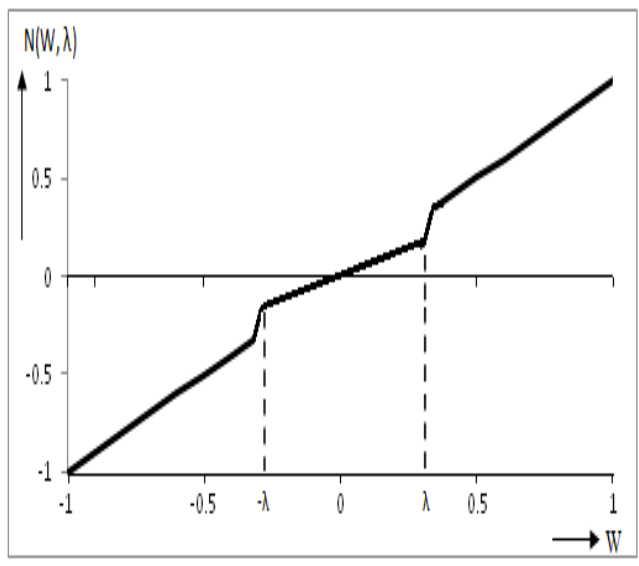

Fig 4: Novel Thresholding Filter

\section{SIMULATION RESULTS AND DISCUSSION}

The results obtained with existing hard, soft, SCAD and novel thresholding functions on noise contaminated image are cited in this section. The Peppers image of size $256 \times 256$ is taken and it is contaminated with additive white Gaussian noise of various standard deviation values. The wavelet used to decompose the noisy image into corresponding wavelet coefficients is coiflet wavelet. These noisy wavelet coefficients are modified using the appropriate thresholding filter function. In thresholding filter the threshold value is fixed using VISU, FDR and translation invariant thresholding methods. The inverse wavelet transform is used to reconstruct the modified noisy wavelet coefficients into denoised image. Quality measure parameters like RMSE and PSNR are used to compare the results.

$$
\begin{aligned}
\mathrm{RMSE} & =\frac{1}{n} \sum_{i=1}^{n}(S(i)-\hat{S}(i))^{2} \\
\mathrm{PSNR} & =10 \log _{10} \frac{\sum_{i=1}^{n} S(i)^{2}}{\sum_{i=1}^{n}(S(i)-\hat{S}(i))^{2}}
\end{aligned}
$$

Where, i represents number of samples, $S(i)$ is original image component and $\hat{S}(i)$ is denoised image component. The simulating process in MATLAB is continuously repeated for about 100 times and then the averages of RMSE and PSNR values are taken.

The same process is implemented on different images then similar type of results will be obtained. The results of image for $\sigma=10,15$ and 20 using hard, soft, SCAD and novel thresholding filters with VISU, FDR and translation invariant method are shown in Table 1-3. The original and denoised image using hybrid thresholding filter with VISU, FDR and translation invariant method are shown in Figures 5-9. Figures 10-15 shows the comparison of the results in VISU, FDR and translation invariant methods.

For a noise standard deviation of $\sigma=10$, RMSE will be 8.4584 and PSNR will be 29.5874 are obtained on denoising the noisy Peppers image with novel thresholding filter using VISU method, the results of RMSE and PSNR obtained for all other thresholding filters are presented in Table 1. The comparison indicates that the novel thresholding filter better denoises the Peppers image than hard, soft and SCAD thresholding filters. The same denoising behavior can be found for $\sigma=15$ and 20 .

Similarly, from the results of RMSE and PSNR for different noise deviations of $\sigma=10,15$ and 20 in Table 2 and Table 3, the newly designed novel filter performs much better than hard, soft and SCAD thresholding filters in denoising the Peppers image using VISU method, FDR method and translation invariant method. 
Table 1 Denoising Results of Peppers Image Using Hard, Soft, SCAD and Novel Thresholding Filters: VISU Method

\begin{tabular}{|c|c|c|c|c|c|c|c|c|c|c|}
\hline & \multicolumn{2}{|c|}{ Noisy Image } & \multicolumn{2}{c|}{ Hard Filter } & \multicolumn{2}{c|}{ Soft Filter } & \multicolumn{2}{c|}{ SCAD Filter } & \multicolumn{2}{c|}{ Novel Filter } \\
\hline & RMSE & PSNR & RMSE & PSNR & RMSE & PSNR & RMSE & PSNR & RMSE & PSNR \\
\hline$\sigma=10$ & 10.0009 & 28.1301 & 10.7203 & 27.5549 & 14.812 & 24.7376 & 14.1872 & 25.103 & 8.4584 & 29.5874 \\
\hline$\sigma=15$ & 14.9916 & 24.6138 & 12.8364 & 25.9662 & 16.757 & 23.6601 & 16.3797 & 23.8519 & 10.38 & 27.8084 \\
\hline$\sigma=20$ & 20.0037 & 22.1086 & 14.7941 & 24.7404 & 19.1339 & 22.4972 & 19.5727 & 22.3042 & 12.2648 & 26.3593 \\
\hline
\end{tabular}

Table 2 Denoising Results of Peppers Image Using Hard, Soft, SCAD and Novel Thresholding Filters: FDR Method

\begin{tabular}{|c|c|c|c|c|c|c|c|c|c|c|}
\hline & \multicolumn{2}{|c|}{ Noisy Image } & \multicolumn{2}{c|}{ Hard filter } & \multicolumn{2}{c|}{ Soft filter } & \multicolumn{2}{c|}{ SCAD filter } & \multicolumn{2}{c|}{ Novel filter } \\
\hline & RMSE & PSNR & RMSE & PSNR & RMSE & PSNR & RMSE & PSNR & RMSE & PSNR \\
\hline$\sigma=10$ & 9.9961 & 28.1342 & 8.5929 & 29.4535 & 11.4405 & 26.9828 & 10.8245 & 27.4528 & 7.3723 & 30.7793 \\
\hline$\sigma=15$ & 15.0181 & 24.5985 & 10.6527 & 27.5825 & 13.1686 & 25.7476 & 12.9888 & 25.869 & 9.6859 & 28.4129 \\
\hline$\sigma=15$ & 19.9881 & 22.1154 & 12.6581 & 26.0879 & 14.5417 & 24.8982 & 14.999 & 24.6157 & 11.6667 & 26.8068 \\
\hline
\end{tabular}

Table 3 Denoising Results of Peppers Image Using Hard, Soft, SCAD and Novel Thresholding Filters: TI Method

\begin{tabular}{|c|c|c|c|c|c|c|c|c|c|c|}
\hline \multirow{2}{*}{} & \multicolumn{2}{|c|}{ Noisy Image } & \multicolumn{2}{c|}{ Hard filter } & \multicolumn{2}{c|}{ Soft filter } & \multicolumn{2}{c|}{ SCAD filter } & \multicolumn{2}{c|}{ Novel filter } \\
\cline { 2 - 11 } & RMSE & PSNR & RMSE & PSNR & RMSE & PSNR & RMSE & PSNR & RMSE & PSNR \\
\hline$\sigma=10$ & 9.994 & 28.136 & 4.7588 & 34.5817 & 8.7162 & 29.3272 & 6.2951 & 32.1601 & 4.739 & 34.8527 \\
\hline$\sigma=15$ & 15.0206 & 24.5971 & 6.3323 & 32.1008 & 10.9964 & 27.3116 & 7.9433 & 30.1329 & 6.2134 & 33.4154 \\
\hline$\sigma=20$ & 20.0701 & 22.1028 & 7.8218 & 30.2657 & 12.5427 & 26.1701 & 10.0191 & 28.1264 & 7.3526 & 31.3216 \\
\hline
\end{tabular}

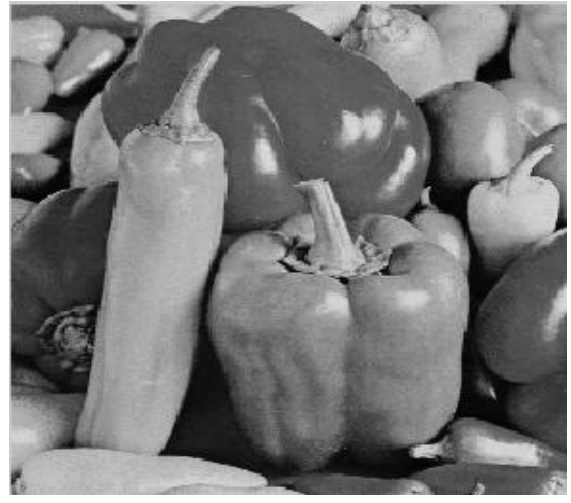

Fig 5: Original Peppers Image

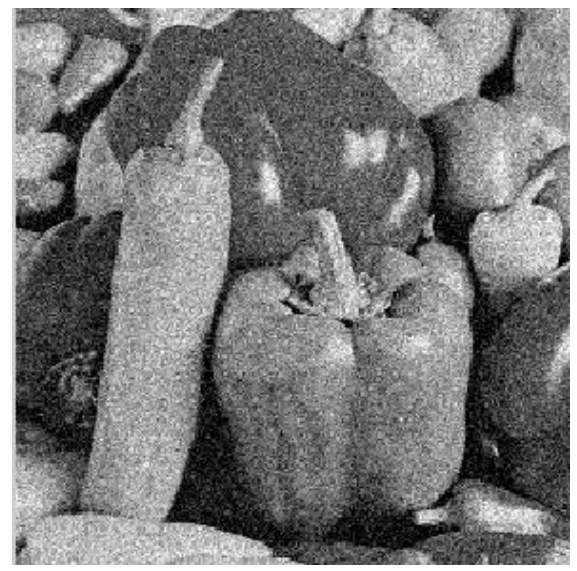

Fig 6: Noisy Peppers Image

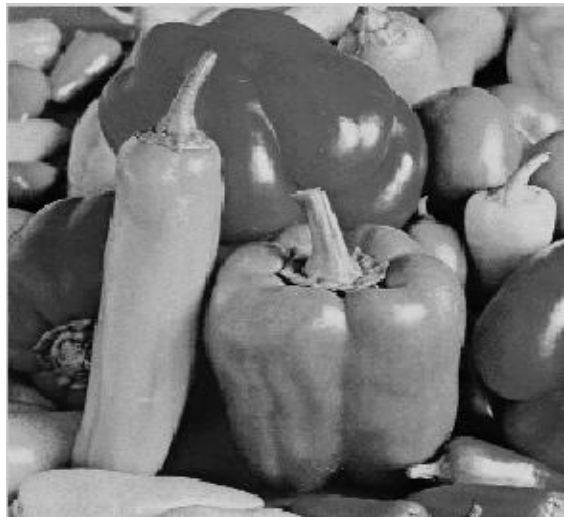

Fig 7: Denoised Peppers Image using novel thresholding filter in VISU method

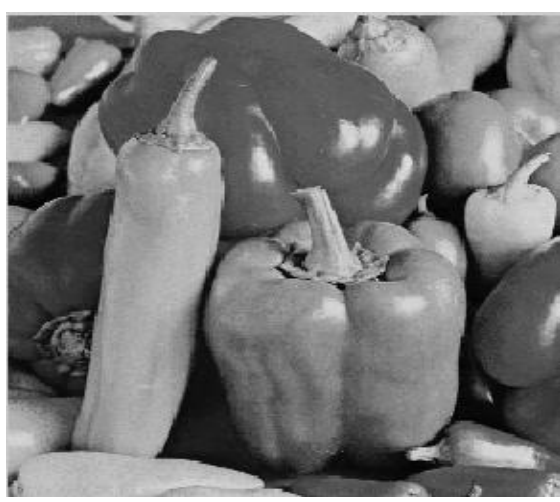

Fig 8: Denoised Peppers Image using novel thresholding filter in FDR method 


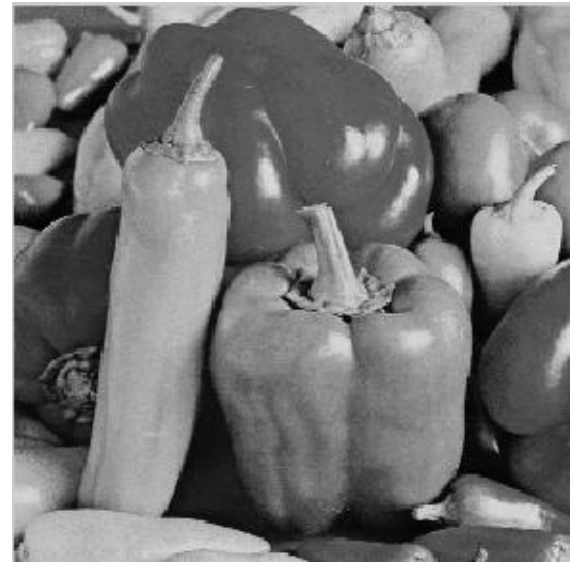

Fig 9: Denoised Peppers Image using novel thresholding filter in TI method

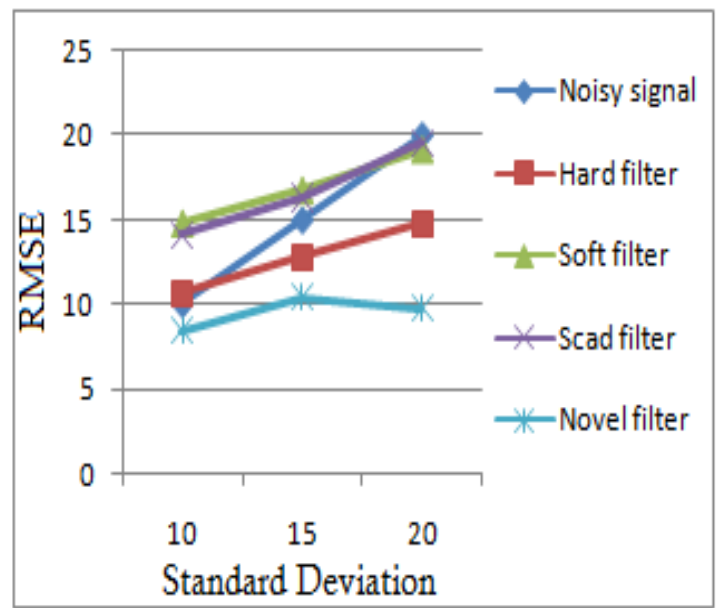

Fig 10: RMSE values of different filters in VISU method.

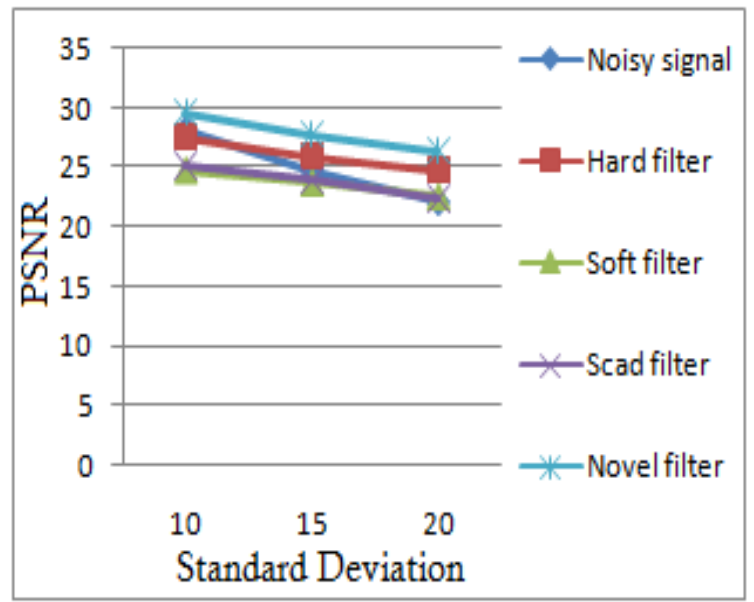

Fig 11: PSNR values of different filters in VISU method.

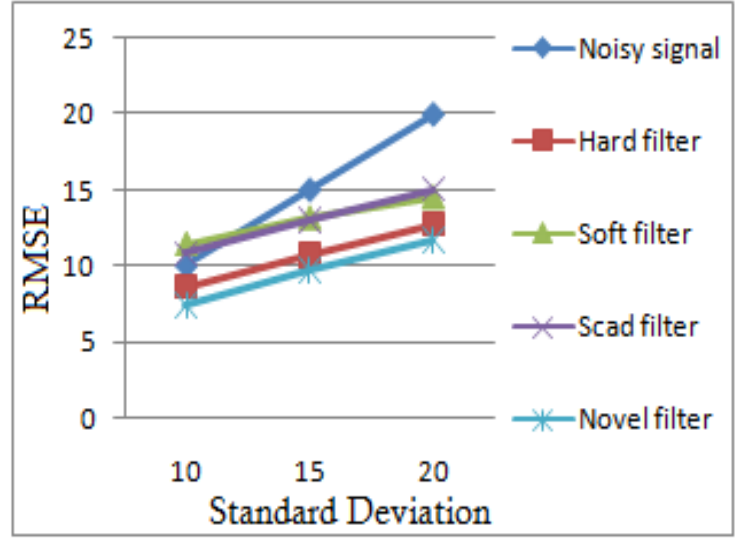

Fig 12: RMSE values of different filters in FDR method.

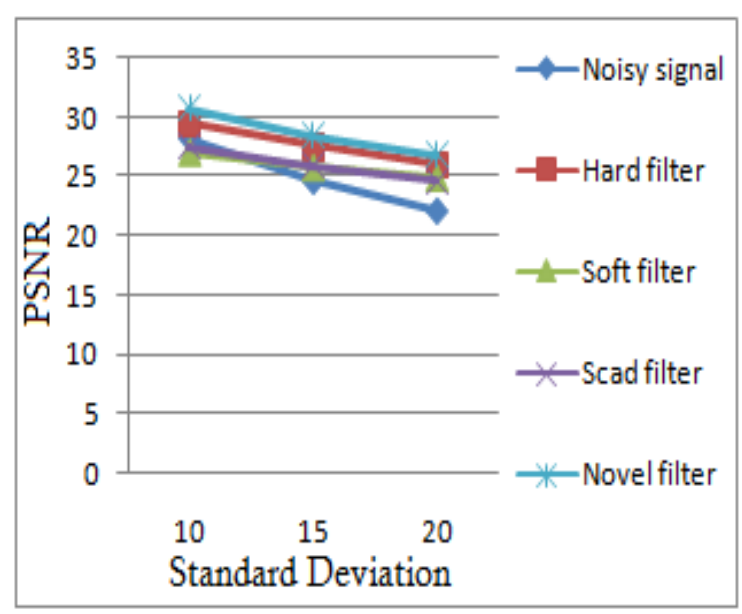

Fig 13: PSNR values of different filters in FDR method.

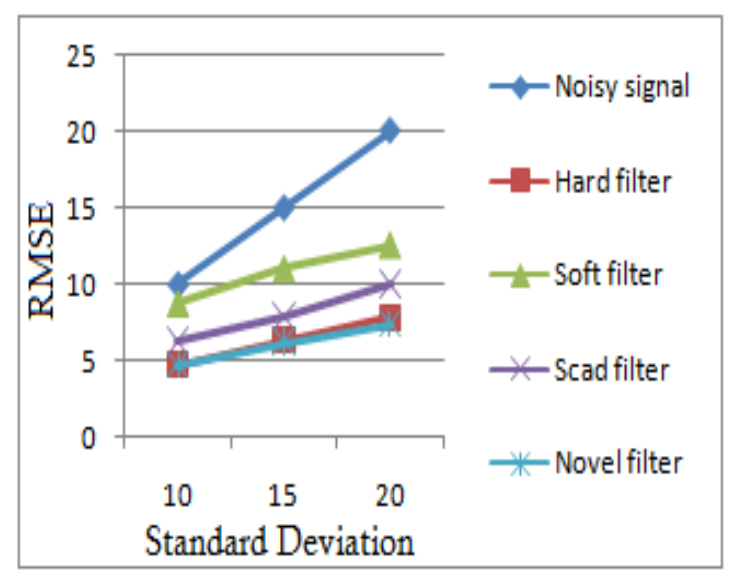

Fig 14: RMSE values of different filters in TI method. 


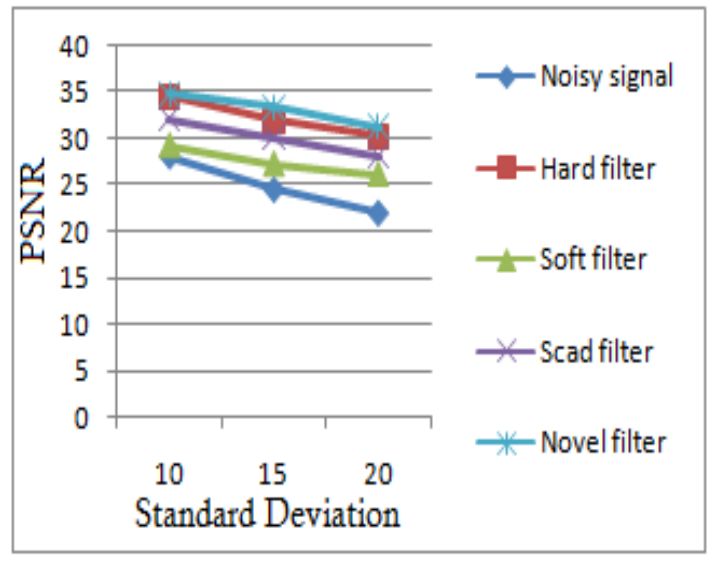

Fig 15: PSNR values of different filters in TI method.

\section{CONCLUSION}

In this paper, a novel thresholding filter for discrete wavelet shrinkage denoising of images is proposed. The performance of this newly designed filter is evaluated by using pepper image. The results obtained are compared with existing hard, soft and SCAD filters. It is found from the results that the novel thresholding filter performs a lot improved than hard, soft and SCAD filters using VISU, FDR and translation invariant methods. From the above three methods TI method is preferred due to its low RMSE and high PSNR for proposed novel filter. In future, this proposed filter is suitable for denoising videos for better quality.

\section{REFERENCES}

[1] Carl Taswell (2000), "The what, how and why of wavelet shrinkage denoising, Computing in Science and Engineering, pp 12-19
[2] Dr. V.V.K.D.V.Prasad (2008), 'Denoising of Biological Signals Using Different Wavelet Based Methods and Their Comparison', Asian Journal of Information Technology, Vol.7, No.4, pp.146-149.

[3] Donoho D, Raimondo M (2004). "Translation Invariant Deconvolution in a Periodic Setting." The International Journal of Wavelets, Multiresolution and Information Processing, 14(1), 415 - 423.

[4] Akhilesh Bijalwan, Aditya Goyal, Nidhi Sethi (2012),'Wavelet Transform Based Image Denoise Using Threshold Approaches', International Journal of Engineering and Advanced Technology (IJEAT), Vol.1, Issue.5,pp.218-221.

[5] Fodor, I. K., and Kamath, C., (2003), "Denoising through wavelet shrinkage: An empirical study", Journal of Electronic Imaging, vol. 12, pp. 151-160.

[6] Jaideva C. Goswami and Andrew K.Chan "Fundamentals of Wavelets", Wiley India Edition, 2009.

[7] Marteen Jansen (2001), "Noise reduction by Wavelet Thresholding," Springer Verlag, Vol. 161.

[8] Donoho, D.L., and Johnstone, I.M., (1995), "Adapting to unknown smoothness via Wavelet Shrinkage", Journal of the American Statistical Association, Vol. 90, No. 432, pp. $1200-1224$

[9] B.Vidakovic and Muller, "Wavelets for kids, a Tutorial Introduction, Wavelet digest" Pearson Education, 1991.

[10] Donoho, D. L., Denoising by Soft Thresholding, IEEE Trans. Information Theory, Vol. 41, No. 3, pp 613-627, 1995. 